\title{
Cooperation and age structure in spatial games
}

\author{
Zhen Wang \\ School of Physics, Nankai University, Tianjin 300071, China and \\ Department of Physics, HongKong Baptist University, Kowloon Tong, HongKong
}

Zhen Wang*

School of Innovation Experiment and School of Software Technology, Dalian University of Technology, Dalian 116024, China

Xiaodan Zhu

School of Innovation Experiment, Dalian University of Technology, Dalian 116024, China

\author{
Jeferson J. Arenzon ${ }^{\dagger}$ \\ Instituto de Física and INCT-Sistemas Complexos, Universidade Federal do Rio Grande do Sul, CP 15051, 91501-970 Porto Alegre RS, Brazil
}

(Received 24 June 2011; revised manuscript received 4 October 2011; published 31 January 2012)

\begin{abstract}
We study the evolution of cooperation in evolutionary spatial games when the payoff correlates with the increasing age of players (the level of correlation is set through a single parameter, $\alpha$ ). The demographic heterogeneous age distribution, directly affecting the outcome of the game, is thus shown to be responsible for enhancing the cooperative behavior in the population. In particular, moderate values of $\alpha$ allow cooperators not only to survive but to outcompete defectors, even when the temptation to defect is large and the ageless, standard $\alpha=0$ model does not sustain cooperation. The interplay between age structure and noise is also considered, and we obtain the conditions for optimal levels of cooperation.
\end{abstract}

DOI: 10.1103/PhysRevE.85.011149

PACS number(s): 02.50.Le, 87.23.-n, 89.65.-s

\section{INTRODUCTION}

The emergence and maintenance of cooperation among self-interested individuals is a challenging problem and an example of a social dilemma in which individual, local interests are inconsistent with collective, global benefits. Studied within the interdisciplinary field of evolutionary game theory [1], this puzzle benefits from techniques borrowed from biology, sociology, economy, computer sciences, and even physics [1-6]. A simple, paradigmatic model to study the problem of cooperation is the prisoner's dilemma (PD) game, which has attracted much attention, both theoretical and experimental [7-12]. In its basic version, two players simultaneously have the choice between cooperation and defection. If both cooperate (defect) they receive the reward $R$ (the punishment $P$ ). If, however, one player chooses cooperation while the other defects, the latter gets the temptation $T$ and the former is left with the sucker's payoff $S$. These payoffs satisfy the ranking $T>R>P>S$ and $2 R>T+S$; thus, defection optimizes the individual payoff, in spite of the fact that mutual cooperation could yield a higher collective benefit. On the other hand, when $T>R>S>P$, the so called snowdrift (SD) game, it is worth it to cooperate, whatever the opponent does, and thus the number of cooperator-defector pairs increases. To overcome this dilemma and avoid exploitation by defectors, several mechanisms have been proposed, among them, network reciprocity in the presence of persistent spatial correlations [13-17].

After the seminal ideas of Axelrod [2] and Nowak and May [13], the role of spatial structure, and its various

\footnotetext{
*wangz@dlut.edu.cn

†arenzon@if.ufrgs.br
}

underlying promoting mechanisms, in evolutionary games have been intensively explored. Among the many identified conditions [5] under which cooperation can be sustained or enhanced, we have the presence of mobile agents [18-22]; heterogeneous activity [23-25] or social diversity [26,27]; complex networks [28-34]. Furthermore, the facilitation effect of reward mechanism [35], partner selection [36], environment influence $[37,38]$, and differences in evolutionary time scales $[39,40]$ have also been identified as boosting mechanisms for cooperation. Other traits may adaptively co-evolve along with the strategy distribution of the population, as the network topology or the evolution rule, which can further enhance cooperation [41-47]. In Ref. [48], the strategy transfer capability was allowed to evolve from a nonpreferential setup, attaining an optimal state that maximizes cooperation. Allowing both the spatial topology and the strategy updating to co-evolve [49,50], cooperation is also largely enhanced.

Perc and Szolnoki [26] introduced a modification on the raw payoff through a random, fixed rescaling factor to take into account extrinsic factors to each agent, finding that some distributions (e.g., power-law) of such factors may greatly promote cooperation. Such a variability may be related to the age structure of a population, affecting individual capabilities when accessing risks, competing, socializing, or generating offspring. Particular examples are reputations or natural and social skills that, on average, increase along lifetime and are reflected on individual fitnesses. In addition, age correlates with body size, which is a dominant factor when an individual tries to impose strategy supremacy during a game.

Although it is undisputable that the age structure is a relevant ingredient when modelling a population, there are, however, several ways in which it may be introduced. On a mean field level, Leslie-type models have been of widespread use, since their introduction in the '40s [51,52], to describe 
well-mixed populations with a (discrete) age structure. Since well-mixed populations of pure strategy (either always or never cooperating) agents are dominated by defectors, the general question of whether cooperation is promoted when age is also taken into account is studied in the context of spatial games, in which strong spatial correlations are relevant. Szolnoki et al. [53] introduced an age-dependent rescaling factor in the strategy transfer capability of agents, showing that cooperators may thrive even when the temptation to defect is large. One important question is how robust and universal are the effects of the age structure on the well-studied spatial version of the PD and SD games. Thus, we consider here a different mechanism, by modulating the raw payoff being accumulated during combats by an age-dependent factor. How strong age influences the outcome of a game depends on the modulation parameter $\alpha$, defined below, and the aim is to study how this mechanism affects the evolution of cooperation. We show, by means of Monte Carlo simulations, that this mechanism can significantly promote cooperation and that intermediate values of $\alpha$ can give optimal levels of cooperation. We explore and interpret this facilitation mechanism and, moreover, examine the impact of different levels of uncertainty by strategy adoption and the possible impact of interaction topology on cooperation. The next section details the model and the implementation of the dynamics. Then, we present our main results and, in the last section, summarize and discuss them.

\section{THE MODEL}

We consider a standard parametrization of the PD game with: temptation to defect $T>1$, reward for mutual cooperation $R=1$, punishment for mutual defection $P=0$, and sucker's payoff $S$ (while $S>0$ is the SD game and $S<0$ is for PD, the interface, at $S=0$, is called the weak version of the PD game). Each player can be either a cooperator (C) or a defector (D), and the initial state is created with equal fractions of each strategy. The contact network is the regular, $L^{2}$ square lattice with nearest-neighbor interactions and periodic boundary conditions; although, for the sake of comparison, we also considered the random regular graph with $z=4$ neighbors per site. Age is included in the model as follows: to each player, at the beginning, is assigned a random integer age $A_{i} \in\left\{0,1, \ldots, A_{\max }\right\}$, where $A_{\max }$ is the maximum possible age. At each Monte Carlo step (MCS), defined as the amount of time in which, on average, each player is updated once, all ages are increased by one. The updating procedure comprises the following elementary steps. First, a randomly chosen agent plays against all its nearest neighbors, earning the raw payoff $p_{i}$. Then, this payoff is rescaled through an age-dependent function:

$$
\Pi_{i}=p_{i}\left(\frac{A_{i}}{A_{\max }}\right)^{\alpha},
$$

where the parameter $\alpha$ determines the level of heterogeneity in the system, that is, how much the heterogeneous age distribution influences the outcome of the game. Then, one of its four neighbors is selected at random and has its rescaled payoff $\Pi_{j}$ evaluated in the same way. At last, player $i$ adopts the strategy $\sigma_{j}$ from the selected player $j$ with the probability

$$
W\left(\sigma_{j} \rightarrow \sigma_{i}\right)=\frac{1}{1+\exp \left[\left(\Pi_{i}-\Pi_{j}\right) / K\right]},
$$

where $1 / K$ is a measure of noise $[54,55]$. After, and only after, one player reaches the maximum age $A_{\max }$, its age is reset to 0 , mimicking a birth-death process. When $\alpha=0$, one recovers the standard $\mathrm{PD}$ game [5], in which age has no effect whatsoever and no payoff rescaling is performed. With finite values of $\alpha$, however, Eq. (1) introduces an obvious demographic heterogeneity into the system during the evaluation process of individual payoffs. When the value of $\alpha$ is large enough, the heterogeneous state will weaken again, because the payoff differences among the majority of players will become very tiny. Notice that, differently from Ref. [53], here the situation is more symmetric since both players influence the transition probabilities through their rescaled payoffs.

The results of Monte Carlo simulations presented below were obtained for lattices with $100^{2}$ to $400^{2}$ individuals, and the average fraction of cooperators $\rho_{\mathrm{C}}$, that is, the number of cooperators divided by $L^{2}$, was determined within the last $10^{4}$ out of the total $2 \times 10^{5}$ MCS. Moreover, the final results were averaged over up to 40 independent runs for each set of parameter values.

\section{RESULTS}

When taking the age structure of the population into account, there is an optimal level of $\alpha$ that, along with the heterogeneous age distribution, maximally enhances cooperation among the individuals. Figure 1 shows how $\rho_{\mathrm{C}}$, the asymptotic fraction of cooperators, depends on the temptation to defect $T$ for different values of $\alpha$ and $S$ both on a square lattice (top 3 rows) and on a random regular graph (bottom row). For all values of $S$ considered and the $\alpha=0$, standard version without age factors, there is not a pure $C$ phase and cooperators go extinct at smaller values of $T$ when compared with $\alpha \neq 0$. For finite $\alpha$, on the other hand, there is a qualitative behavioral change. For moderate $\alpha$ ( 1 and 2 in the figure), cooperators are not only able to survive over a larger interval of $T$ but may even reach near-complete dominance status $\left(\rho_{\mathrm{C}}=1\right)$. Nonetheless, on further increasing $\alpha$ (say, 20) the level of cooperation decreases once again. Thus, moderate values of $\alpha$ allow cooperators to better thrive, optimally enhancing cooperation, a phenomenon that is analogous to the so-called coherence resonance [56,57]. Notice also that persistent neighbors (existing both in the square lattice and in the random regular graph) are essential to sustain cooperation, while in the absence of correlations between neighbors (as in a well-mixed population), defectors dominate. This information is summarized in the phase diagram shown in Fig. 2 (since the overall behavior is rather similar, we herein focus on the $S=0$ case on a square lattice). The threshold value of $T$ (the above curve) marks the extinction of cooperators and the transition between the coexisting $(C+D)$ and the pure $D$ phases. Notice that the initial increase, from $\alpha=0$ toward the maximum cooperation at $\alpha \simeq 2.5$ is fast but, in contrast, the subsequent decrease after the maximum is much slower. There is a second transition line, separating the coexistence phase, 


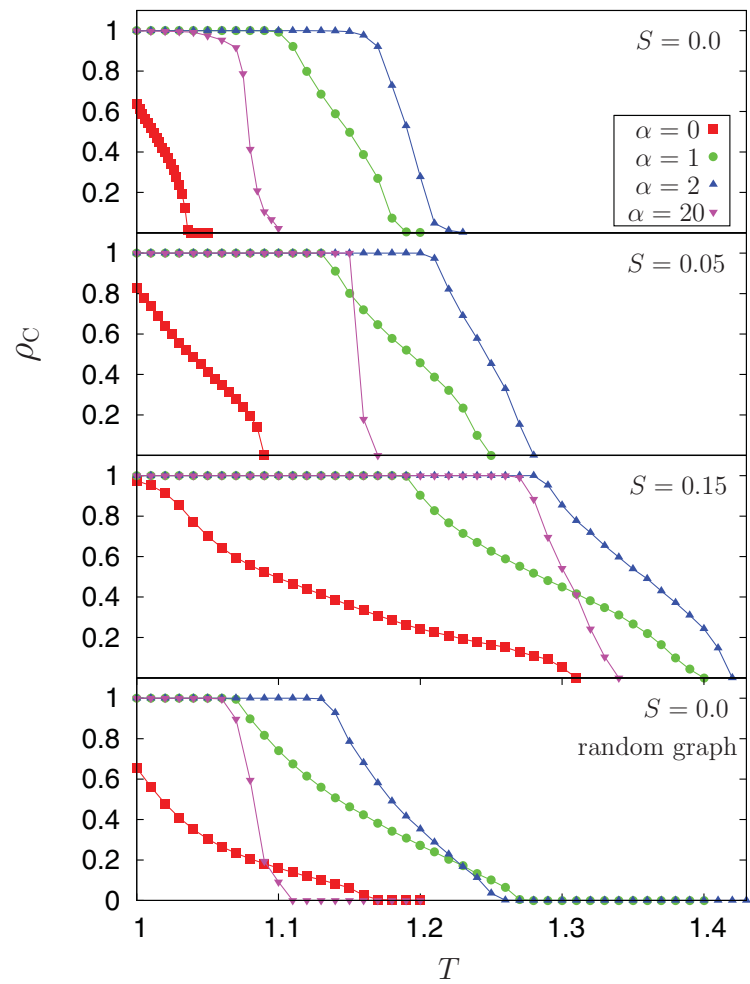

FIG. 1. (Color online) Asymptotic fraction of cooperators $\rho_{\mathrm{C}}$ as a function of the temptation $T$ for different values of $\alpha$ and $S$, with $K=0.1$ and $A_{\max }=100$. The topmost row, with $S=0$, is the weak PD game while the second and third row have $S>0$, that is, the SD game. Since for higher values of $S$ it is better, against a defector, to cooperate, the overall increase in cooperation is not a surprise. Notice that for the original model, $\alpha=0$, cooperation vanishes for not too large temptations and cooperators never fully occupy the system, $\rho_{\mathrm{C}}<1$. For finite $\alpha$ and small values of $T$, cooperators fully invade the system and there is an optimum $\alpha$ for which cooperation is enhanced. Apart the crossing between some of the curves for $S>0$, the overall behavior is the same in all cases. The last row presents results for the weak PD on a random regular graph (the previous figures were for the square lattice). Cooperation is enhanced when compared with the original $\alpha=0$ case as well, showing that persistent neighbors (in contrast to well-mixed ones) is essential, while the small loops present in the square lattice, but absent in the random graph, do not qualitatively change the picture.

which is quite narrow, from the pure $\mathrm{C}$ phase, whose behavior roughly follows the upper line.

Figure 3 shows the temporal evolution of the density of cooperators, $\rho_{\mathrm{C}}(t)$, for several values of $\alpha$ and $T=1.125$ in order to help understand the role of spatial heterogeneities in promoting cooperation. Unless cooperators are organized in compact clusters, defectors will easily predate them. Indeed, soon after the start, since the initial state is randomly chosen, defectors are more successful and the number of cooperators in the population decreases. This is a general feature of the PD game in spatially structured environments. However, different values of $\alpha$ will result in diverse trends at large times. For the above parameters, the standard, $\alpha=0$ case, does not form clusters strong and fast enough, leading to a fast extinction of cooperators. Similarly, for large enough $\alpha$ (e.g., 20), cooperators do not survive either, though it takes them a

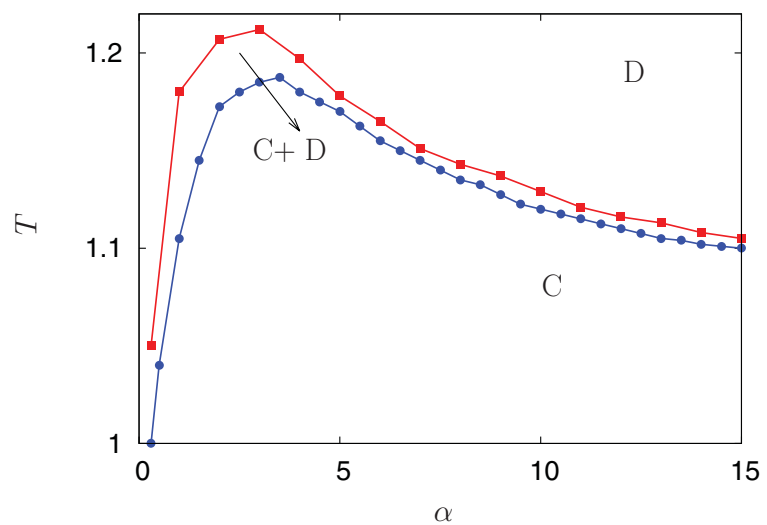

FIG. 2. (Color online) Phase diagram showing the critical threshold (red box symbols) above which cooperators go extinct, along with the pure $\mathrm{C}$ and coexisting phases. As $\alpha$ increases, there is at first a fast increase both in the cooperators dominated phase and in the coexistence one. After the optimal cooperation happening at $\alpha \simeq 2.5$, further increasing $\alpha$ is detrimental for cooperation, although the decrease is slow.

long time to die out. For intermediate values of $\alpha$, surprisingly, the initial downfall of cooperators is halted, allowing for the subsequent fast spreading of cooperation to complete or near complete dominance, as shown for $\alpha=1$ and 2. Moreover, the sooner the initial decrease of cooperators is halted, the faster the recovery of cooperation will be, leading to a larger value of $\rho_{\mathrm{C}}$. Thus, we argue that for moderate values of $\alpha$, a recovery mechanism is in course, halting and eventually reversing the decrease of cooperation. Indeed, by measuring the persistence $P(t)$, that is, the fraction of sites that did not change strategy

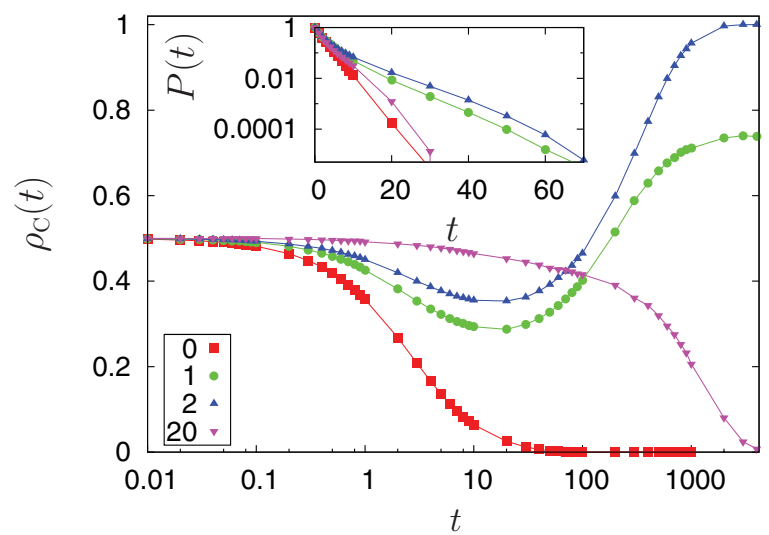

FIG. 3. (Color online) Time evolution of cooperation for different values of $\alpha, T=1.125, S=0$, and $K=0.1$. Since the initial state is random and cooperators are not yet organized in compact groups, many of them are easy prey for defectors and there always is an initial decrease on their number. For $\alpha=0$ and 20, cooperators do not recover from the initial downfall, the curves are monotonously decreasing and they get extinct. Inset: the fraction of agents that did not change strategy up to the time $t$ [the persistence $P(t)$ ]. Notice that for $\alpha=0$ the decay is exponential while it does become stretched for $\alpha \neq 0$. Moreover, for the cases presenting cooperation at long times ( $\alpha=1$ and 2), the persistence decays more slowly, showing that the initially formed clusters are more resistent (the long lasting agents are cooperators). 

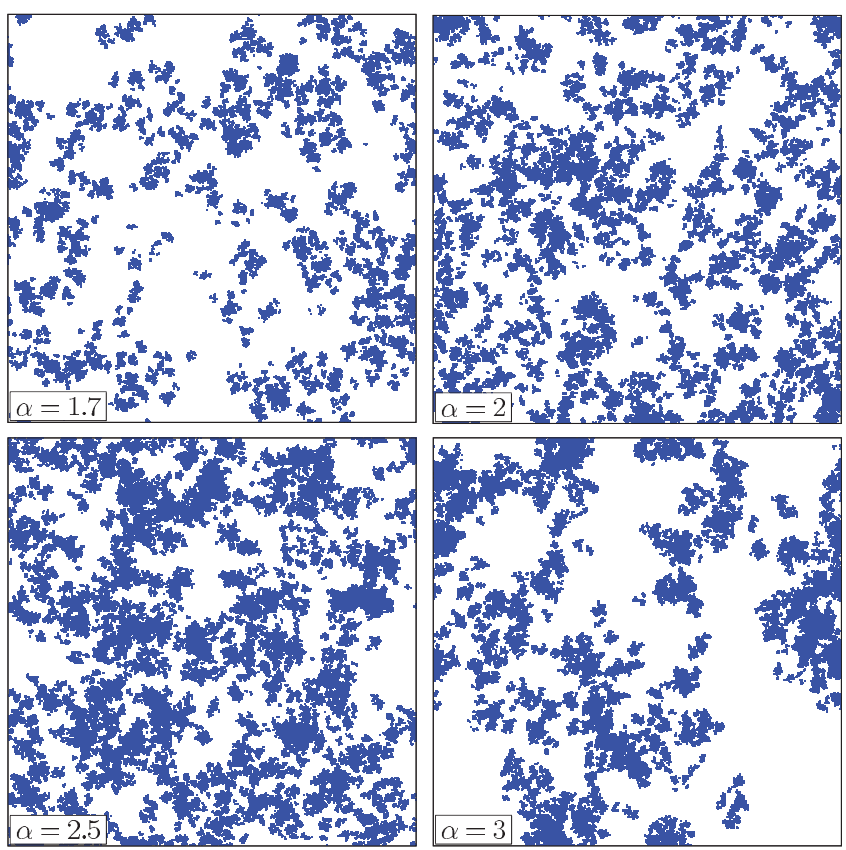

FIG. 4. (Color online) Lattice snapshots for different values of $\alpha$ on a $400^{2}$ square lattice, $S=0, T=1.2$, and $K=0.1$, showing the clusterized cooperators after $10^{5} \mathrm{MCS}$.

up to time $t$, one notices that in those cases where cooperation survives ( $\alpha=1$ and 2), the decay of $P(t)$ is much slower when compared to the $\alpha=0$ and 20 cases, in which the decay is almost exponential. In particular, the long time tail of $P(t)$ corresponds to enduring cooperators.

It is also instructive to analyze typical spatial configurations of cooperators and defectors for different values of $\alpha$, as shown in Fig. 4 for $T=1.2$ and $K=0.1$. Notice that for these parameters, the original, $\alpha=0$ case does not sustain cooperation and coexistence appears on a finite interval of $\alpha$ $(\mathrm{C}+\mathrm{D}$ phase) in which highly clustered cooperators survive the exploitation by defectors [58]. The amount of cooperators is correlated with the geometric properties of these clusters. Indeed, it can be noticed that the average cluster size increases with $\alpha$, Fig. 5, and the maximum level of cooperation corresponds to the maximum average size (since clusters

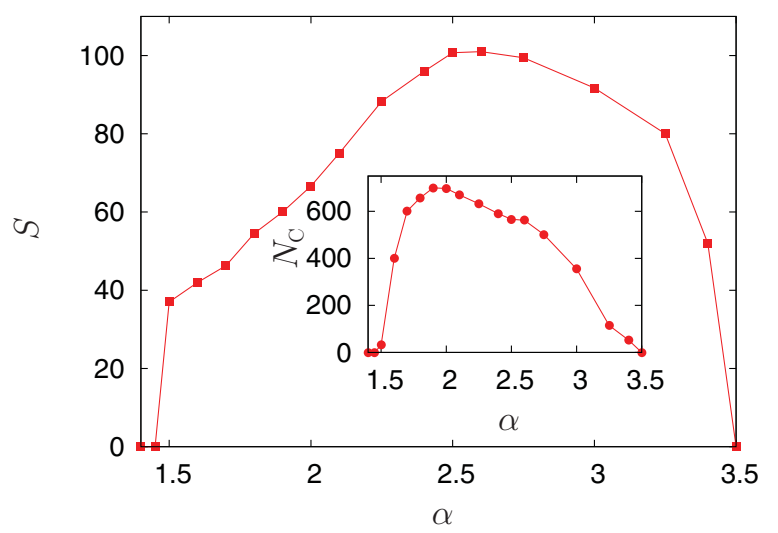

FIG. 5. (Color online) Stationary average size of cooperators clusters for a $400^{2}$ lattice with $T=1.2, S=0$, and $K=0.1$. Inset: corresponding number of cooperators clusters, $N_{\mathrm{C}}$. merge as they grow, the number of cooperators clusters has its maximum at a smaller value of $\alpha$, as shown in the inset of Fig. 5). Notice that these average properties of clusters are stationary, albeit individual clusters keep evolving. For the chosen value of $T$, the system does not attain complete cooperation (see the phase diagram in Fig. 2), but for a slightly smaller $T$, the pure $\mathrm{C}$ phase would be crossed. In this case, since cooperation dominates, a single cluster survives. It has been shown $[25,30]$ that heterogeneity favors the spreading of cooperation which, to some extent, explains the promotion effect observed here. According to Eq. (1), large enough values of $\alpha$ will lead to a heterogeneous distribution within the system but also reduce the heterogeneous state compared with that of smaller $\alpha$, since payoff difference among majority of players will become very tiny. Hereby, these patterns suggest that heterogeneity is significant but not sufficient to explain the promotion phenomenon for moderate $\alpha$.

An important remaining question concerns how robust the above effects are in the presence of noise in the process of strategy adoption Eq. (2). Figure 6 shows the $K-T$ phase diagrams for $\alpha=0,2$, and 20 (from top to bottom). The original, $\alpha=0$ case [56], in addition to the monotonous

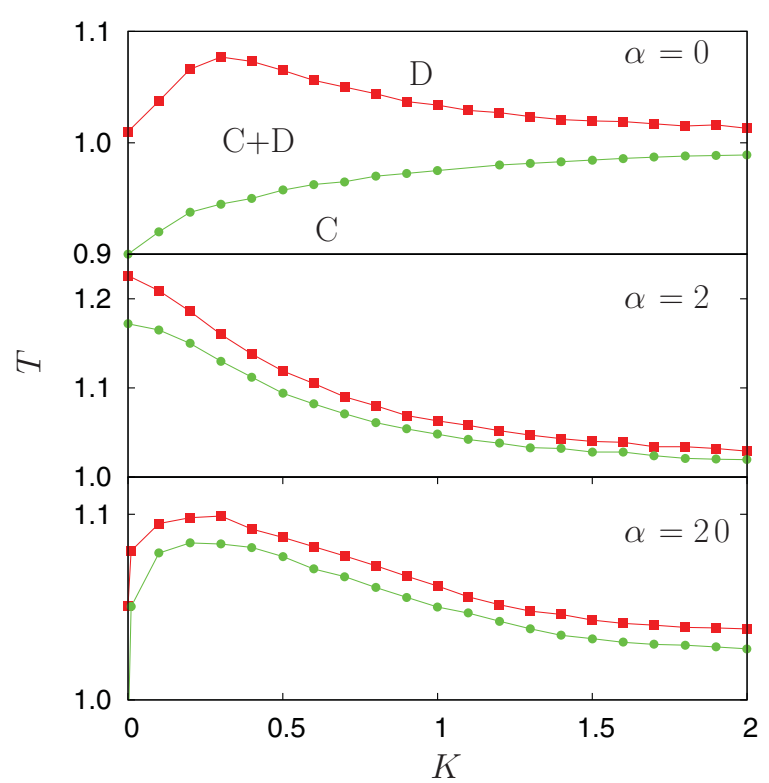

FIG. 6. (Color online) Full $K-T$ phase diagram for $\alpha=0,2$, and 20 and $S=0$. Green circles and red boxes mark the border between pure $C$ and $D$ phases and the mixed $C+D$ phase, respectively. For $\alpha=0$ (top panel) [56], the presence of some uncertainty in the strategy dynamics $(K>0)$ leads to an optimal level of cooperation (the upper curve has a maximum at $K \simeq 0.3$ ). For small $\alpha$ (middle panel), the optimal noise threshold shifts toward zero but, when compared with the $\alpha=0$ case, is larger for all values of $K$. Upon further increasing $\alpha$, as shown in the bottom panel, the system becomes once again more noise tolerant. However, there is a huge difference between all these cases: the pure $\mathrm{C}$ phase is greatly enhanced at the expense of the coexistence region whenever $\alpha \neq 0$. Indeed, only for $\alpha=0$ the $\mathrm{C}+\mathrm{D}$ regions is prominent. Moreover, the large $\alpha$ case also presents an optimal value of $T$ for the transition between the pure and mixed phases $(K \simeq 0.25)$. Thus, by adjusting the level of heterogeneity one can either increase the amount of cooperation $(\operatorname{small} \alpha)$ or the robustness to noise (large $\alpha$ ). 
increasing border between the pure $\mathrm{C}$ and mixed $(\mathrm{C}+\mathrm{D})$ phases, presents a maximum, around $K \simeq 0.3$, in the line separating the mixed and pure $\mathrm{D}$ phases. Thus, in this case, a finite amount of noise increases the presence of cooperators in the population. The introduction of age factors, through $\alpha>0$, drastically changes this scenario. Whenever $\alpha>0$, there is a large increase in the pure $\mathrm{C}$ phase, its border now lying mostly in the $T>1$ region for all values of $K$ (middle and bottom panels in Fig. 6). Notice that for all cases presented, the monotonicity of the pure $\mathrm{C}$ phase border strongly differ: for $\alpha=0,2$, and 20, respectively, it is ever increasing, ever decreasing, and nonmonotonous. Indeed, the fully cooperative phase has its maximum for $K \gg 1$, $K \rightarrow 0$, and $K \simeq 0.3$ for these values of $\alpha$. However, even if intermediate values of $\alpha$ have decreasing levels of cooperation as the noise increases, it is not less noise tolerant than the other reported values of $\alpha$ since cooperators are able to resist in more severe conditions (larger temptation). For example, compare the values of $K$ around which $\alpha=0$ and 20 present its maxima, $K \sim 0.25-0.3$ : the maximum value of $T$ below which cooperation exists is substantially larger for $\alpha=2$. It has been conjectured that an optimal uncertainty for the evolution of cooperation only occurs on interaction graphs lacking a percolating cluster of overlapping triangles [5]. Therefore, if the conjecture proves to be valid, the phase diagrams of Fig. 6 seem to indicate that for moderate values of $\alpha$, there is a change in the effective interaction topology: previously disconnected triplets (triangles are absent in the square lattice) may now become effective triangles once their connections are enhanced by $\alpha$. A similar phenomenon has been observed in public goods games [59] as well. It would be interesting to further investigate the structure of such effective topology.

\section{CONCLUSION}

We considered both the prisoner's dilemma and the snowdrift game, standard models for cooperation, in an agestructured population in which the final payoff is directly correlated to the age. Although the raw payoff obtained after interacting with other agents depends only on the strategies of both opponents, we consider here that the older the agent is, the larger the fraction of the raw payoff that is actually earned (the efficiency of an agent grows as it gets older and more experienced). This discount parameter depends on the parameter $\alpha$ through Eq. (1), which controls how the heterogeneous distribution of ages (births are not synchronized) throughout the population will be reflected on the payoff distribution. The larger the $\alpha$, the stronger the effects of age, with the age-independent case being recovered for $\alpha=0$. For moderate values of $\alpha$, cooperation is either enhanced or allowed in regions where the pure model is dominated by defectors. Extensive simulations were performed in order to determine how the behavior depends on $\alpha$ and on the noise $K$ and, moreover, to give a geometric interpretation for the results based on the spatial distribution of cooperators. In particular, the latter also improves the amount of cooperation even further when compared to the optimal level for $\alpha=0$. Persistent neighbors are necessary in order to have cooperation in this model (the well-mixed, mean-field case does not support a finite fraction of cooperators), although the presence of small loops significantly increases the amount of cooperators (in the square lattice, loops are small since the nearest neighbors of a given site are connected through a common neighbor, while in the random graph, loops are very large and thus unimportant). The promotion of cooperation is also associated with a recovery effect. Indeed, for intermediate values of $\alpha$, the initial time downfall of cooperators is hindered, helping cooperators to form more robust clusters that are impervious to defectors' exploitation.

The above results help in building a comprehensive understanding of the role of age in a simple framework upon which a co-evolutionary model can be built. Although we take the simplest version here in which the lifespan $A_{\max }$ and $\alpha$ are not traits, it is possible to have them co-evolving with the strategies. Moreover, since age-related phenomena are ubiquitous, it is an important ingredient to be included in more realistic models of populations, in particular when other cooperation mechanisms (e.g., kinship selection) are involved.

\section{ACKNOWLEDGMENTS}

Zhen Wang acknowledges support from the Center for Asia Studies of Nankai University (Grant No. 2010-5) and from the National Natural Science Foundation of China (Grant No. 10672081). J.J.A. was partially supported by the Brazilian agencies CNPq (PROSUL-490440/2007), CAPES, and PRONEX/Fapergs.
[1] J. Maynard Smith, Evolution and the Theory of Games (Cambridge University Press, Cambridge, 1982).

[2] R. Axelrod, The Evolution of Cooperation (BasicBooks, New York, 1984).

[3] M. A. Nowak, Evolutionary Dynamics: Exploring the Equations of Life (Belknap Press, Cambridge, 2006).

[4] M. A. Nowak, Science 314, 1560 (2006).

[5] G. Szabó and G. Fath, Phys. Rep. 446, 97 (2007).

[6] C. P. Roca, J. A. Cuesta, and A. Sanchez, Phys. Rev. E 80, 046106 (2009).

[7] B. Kerr, M. A. Riley, M. W. Feldman, and B. J. M. Bohannan, Nature (London) 418, 171 (2002).
[8] J. M. Pacheco, A. Traulsen, and M. A. Nowak, Phys. Rev. Lett. 97, 258103 (2006).

[9] J. Poncela, J. Gomez-Gardeñes, L. M. Floria, and Y. Moreno, New J. Phys. 9, 184 (2007).

[10] D. Helbing, A. Szolnoki, M. Perc, and G. Szabó, PLoS Comput. Biol. 6, e1000758 (2010).

[11] A. Traulsen, D. Semmann, R. D. Sommerfeld, H.-J. Krambeck, and M. Milinski, Proc. Natl. Acad. Sci. USA 107, 2962 (2010).

[12] J. Grujić, C. Fosco, L. Araujo, J. A. Cuesta, and A. Sánchez, PLoS ONE 5, e13749 (2010).

[13] M. A. Nowak and R. M. May, Nature (London) 246, 15 (1992).

[14] N. Masuda and K. Aihara, Phys. Lett. A 55, 313 (2003). 
[15] S. Wang, M. S. Szalay, C. Zhang, and P. Csermely, PLoS ONE 3, e1917 (2008).

[16] W.-B. Du, X.-B. Cao, and M.-B. Hu, Europhys. Lett. 87, 60004 (2009).

[17] Z. Wang and M. Perc, Phys. Rev. E 82, 021115 (2010).

[18] M. H. Vainstein, A. T. C. Silva, and J. J. Arenzon, J. Theor. Biol. 244, 722 (2007).

[19] D. Helbing and W. Yu, Proc. Natl. Acad. Sci. USA 106, 3680 (2009).

[20] S. Meloni, A. Buscarino, L. Fortuna, M. Frasca, J. GomezGardenes, V. Latora, and Y. Moreno, Phys. Rev. E 79, 067101 (2009).

[21] Z.-X. Wu and P. Holme, Phys. Rev. E 80, 026108 (2009).

[22] L.-L. Jiang, W.-X. Wang, Y.-C. Lai, and B.-H. Wang, Phys. Rev. E 81, 036108 (2010).

[23] A. Szolnoki and G. Szabó, Europhys. Lett. 77, 30004 (2007).

[24] M. Perc and Z. Wang, PLoS ONE 5, e15117 (2010).

[25] G. Szabo and A. Szolnoki, Phys. Rev. E 79, 016106 (2009).

[26] M. Perc and A. Szolnoki, Phys. Rev. E 77, 011904 (2008).

[27] F. C. Santos, M. D. Santos, and J. M. Pacheco, Nature (London) 454, 213 (2008).

[28] J. Poncela, J. Gomez-Gardeñes, L. M. Floria, A. Sánchez, and Y. Moreno, PLoS ONE 3, e2449 (2008).

[29] S. Assenza, J. Gomez-Gardenes, and V. Latora, Phys. Rev. E 78, 017101 (2008).

[30] F. C. Santos and J. M. Pacheco, Phys. Rev. Lett. 95, 098104 (2005).

[31] W.-B. Du, X.-B. Cao, L. Zhao, and M.-B. Hu, Physica A 388, 4509 (2009).

[32] Z. Rong, X. Li, and X. Wang, Phys. Rev. E 76, 027101 (2007).

[33] H. Ohtsuki, C. Hauert, E. Lieberman, and M. A. Nowak, Nature (London) 441, 502 (2006).

[34] S. Lozano, A. Arenas, and A. Sánchez, PLoS ONE 3, e1892 (2008).

[35] R. Jiménez, H. Lugo, J. A. Cuesta, and A. Sánchez, J. Theor. Biol. 250, 475 (2008).

[36] F. Fu, C. Hauert, M. A. Nowak, and L. Wang, Phys. Rev. E 78, 026117 (2008).
[37] Z. Wang, A. Murks, W.-B. Du, Z.-H. Rong, and M. Perc, J. Theor. Biol. 277, 19 (2011).

[38] Z. Wang, W.-B. Du, X.-B. Cao, and L.-Z. Zhang, Phys. A 390, 1234 (2010).

[39] C. P. Roca, J. A. Cuesta, and A. Sanchez, Phys. Rev. Lett. 97, 158701 (2006).

[40] Z.-X. Wu, Z. Rong, and P. Holme, Phys. Rev. E 80, 036106 (2009).

[41] M. Perc and A. Szolnoki, BioSystems 99, 109 (2010).

[42] M. G. Zimmermann and V. M. Eguíluz, Phys. Rev. E 72, 056118 (2005).

[43] W. Li, X.-M. Zhang, and G. Hu, Phys. Rev. E 76, 045102(R) (2007).

[44] H. Ebel and S. Bornholdt, Phys. Rev. E 66, 056118 (2002).

[45] J. Poncela, J. Gomez-Gardeñes, A. Traulsen, and Y. Moreno, New J. Phys. 11, 083031 (2009).

[46] A. Yamauchi, J. Tanimoto, and A. Hagishima, BioSystems 103, 85 (2011).

[47] F. C. Santos, J. M. Pacheco, and T. Lenaerts, Proc. Natl. Acad. Sci. USA 103, 3490 (2006).

[48] A. Szolnoki and M. Perc, New J. Phys. 10, 043036 (2008).

[49] J. Poncela, J. Gomez-Gardeñes, L. M. Floria, Y. Moreno, and A. Sánchez, Europhys. Lett. 88, 38003 (2009).

[50] M. G. Zimmermann, V. M. Eguíluz, and M. SanMiguel, Phys. Rev. E 69, 065102(R) (2004).

[51] B. Charlesworth, Evolution in Age-Structured Populations (Cambridge University Press, Cambridge, 1994).

[52] M. B. Usher and M. H. Williamson, Biometrics 26, 1 (1970).

[53] A. Szolnoki, M. Perc, G. Szabo, and H.-U. Stark, Phys. Rev. E 80, 021901 (2009).

[54] L. E. Blume, Games Econ. Behav. 5, 387 (1993).

[55] G. Szabo and C. Toke, Phys. Rev. E 58, 69 (1998).

[56] M. Perc, New J. Phys. 8, 22 (2006).

[57] A. Traulsen, T. Röhl, and H. G. Schuster, Phys. Rev. Lett. 93, 028701 (2004).

[58] C. Hauert and M. Doebeli, Nature (London) 428, 643 (2004).

[59] A. Szolnoki, M. Perc, and G. Szabo, Phys. Rev. E 80, 056109 (2009). 